\section{Prevalência do uso de produtos derivados do tabaco e fatores associados em mulheres no Paraná, Brasil}

\author{
Prevalence of tobacco use and associated factors \\ among women in Paraná State, Brazil
}

\author{
1 University of Alabama at \\ Birmingham, Birmingham, \\ U.S.A. \\ 2 University of Massachusetts, \\ Worcester, U.S.A. \\ 3 Pontifícia Universidade \\ Católica do Paraná, Curitiba \\ Brasil. \\ Correspondência \\ L. Bittencourt \\ University of Alabama at \\ Birmingham. \\ Medical Towers 101, \\ 1717 11th Avenue South, \\ Birmingham, Alabama \\ 35205, U.S.A. \\ LGoncalves@mail.dopm.uab.edu
}

\begin{abstract}
This study aimed to estimate the prevalence of tobacco use and to describe the demographic profile of female smokers in Paraná State, Brazil. The study used a cross-sectional populationbased design with cluster sampling $(n=2,153)$ of women 18 years or older in seven cities. Prevalence of smoking was $13.4 \%$, ranging from $10 \%$ in Cascavel to $19 \%$ in Irati. According to multivariate analysis, city of residence, marital status, and schooling were significantly associated with tobacco use. Women in Irati $(\mathrm{OR}=2.08$; 95\% CI: 1.22-3.54) were more likely to smoke than those in Cambé. Married women and widows were less likely to smoke (OR =0.47; 95\%CI: 0.30-0.73 and $O R=0.43$; 95\%CI: 0.22-0.87) than single women . Women living with a partner (but not married) were more likely to smoke than single women $(O R=2.49 ; 95 \% C I: 1.12-5.53)$, and women with university degrees were less likely to smoke than those with eight years of school or less $(O R=0.41$; 95\%CI: 0.22-0.87). The results confirm the need for tobacco control programs that take gender and regional differences into account.
\end{abstract}

Tobacco; Smoking; Women
Isabel C. Scarinci 1 Lorna Bittencourt 1 Sharina Person 2 Regina C. Cruz 3 Simone Tetu Moysés 3

\section{Introdução}

O uso do tabaco, em todas as suas formas, é identificado como um importante problema de saúde pública e está associado a doenças cardiovasculares, respiratórias, neoplasias e é considerado a principal causa de mortes evitáveis $1,2,3,4$. As previsões são de que em 2030, caso não haja nenhuma mudança em relação à restrição de seu uso, mais de 8 milhões de pessoas irão morrer por ano com problemas de saúde associados ao uso de produtos derivados do tabaco 1,4 .

O uso do tabaco também é responsável por um impacto econômico substancial no mundo por conta da sua relação com a diminuição de mão de obra e gastos com a saúde. Isto ocorre devido às altas taxas de morbidade e mortes prematuras associadas ao uso do tabaco, assim como gastos em assistência médica 5 . No Brasil, 7,7\% dos custos pagos pelo Sistema Único de Saúde (SUS) relacionados a internações e procedimentos quimioterápicos estão relacionados ao uso do tabaco ${ }^{6}$.

Alguns estudos e medidas de saúde pública já estão sendo realizados no Brasil a fim de documentar e/ou controlar o uso do tabaco 7,8,9,10,11. Em 2008, a prevalência de uso de qualquer produto derivado do tabaco no Brasil foi de $17,2 \% 12$. Na Região Sul, responsável por 95\% da produção do tabaco no país, a prevalência foi ainda maior (19\%) 12,13. Nesta pesquisa, o Paraná foi o oitavo estado com maior prevalência de fuman- 
tes no Brasil $(18,4 \%)$ 12. Porém, há uma escassez de estudos buscando entender as questões de gênero e tabaco, especialmente em mulheres. A Convenção-Quadro para Controle do Tabaco, o primeiro tratado internacional baseado em evidência que visa engajar o mundo num esforço global para o controle do tabaco, tem como um dos seus princípios norteadores " a necessidade de criar medidas específicas para trabalhar os fatores de risco relacionados às questões de gênero, quando forem desenvolvidas estratégias para o controle do tabaco" 1 (p. 6).

A prevalência do uso do tabaco em mulheres no Brasil tem sido relativamente baixa $(13,1 \%)$, quando comparada aos homens $(21,6 \%)$, e esta prevalência tem diminuído nos últimos anos 12,14,15. Entretanto, podemos identificar quatro grandes fatores que indicam a necessidade de estudos regionais que propiciem um entendimento mais aprofundado da prevalência e fatores associados ao uso do tabaco em mulheres. Primeiro, a observação de importante disparidade em termos de prevalência nas diferentes regiões do Brasil, com a Região Sul tendo a maior prevalência de mulheres usuárias do tabaco (15,9\%) 12.

Segundo, a iniciação entre jovens do sexo feminino também apresenta grandes diferenças regionais. Em uma pesquisa realizada em 20022003 com jovens em escolas de 12 capitais brasileiras foi verificado que a experimentação do cigarro era maior entre meninos do que entre meninas, exceto para as capitais do Sul do país, em que a experimentação era maior entre as meninas do que entre os meninos $(51 \%$ e $39 \%$ respectivamente em Curitiba, Paraná) 9 . O mesmo padrão foi observado em relação aos atuais fumantes (que fumaram um ou mais cigarros nos últimos 30 dias): $16 \%$ entre meninas e $11 \%$ entre meninos em Curitiba 16. Outra pesquisa ampla desenvolvida em 107 cidades brasileiras mostrou que meninas entre 12 e 17 anos de idade tendem a relatar que fumam há mais tempo e com maior frequência do que meninos da mesma faixa etária (16,2\% e 15,2\% respectivamente) 17 .

Terceiro, ainda que alguns estudos não sejam convergentes $18,19,20,21,22,23$, a literatura sugere que mulheres são menos propensas a deixar de fumar do que homens, embora elas fumem menos que os homens $24,25,26$. De fato, na pesquisa de 2002-2003 realizada em 15 capitais brasileiras, foi observado que a cessação do uso do tabaco nas três capitais do Sul do país era maior entre homens do que entre as mulheres $(48,7 \%$ e $42,4 \%$ respectivamente, em Curitiba) 9 .

E quarto, as companhias do tabaco têm concentrado esforços para promover o uso de cigarros entre mulheres, “...usando imagens sedutoras, porém falsas, que remetem à vitalidade, pessoa magra, modernidade, emancipação, sofisticação e apelo sexual" 27 (p. 7). Revendo documentos da indústria tabaqueira sobre a América Latina e Caribe, Bialous \& Shatenstein 28 revelam suas táticas específicas para mulheres, considerando questões culturais e de gênero. Algumas das razões pelas quais o Virginia Slims foi lançado na América Latina estão associadas à argumentação de que "imagem e beleza são elementos fortes na sociedade latino-americana; as mulheres têm papéis distintos e diferenciados dos homens na sociedade latino-americana; mulheres latinoamericanas tendem a ser muito envolvidas pela moda; mulheres latino-americanas parecem confortáveis com o conceito de feminilidade" 28 (p. 65). Também advogam a "necessidade de liberdade e independência” 29 (p. 27) entre mulheres, particularmente em um país como o Brasil, onde as mulheres estão lutando pela igualdade de gênero 29,30.

Tendo em vista esta necessidade de entender as peculiaridades regionais do uso do tabaco em mulheres, o objetivo deste estudo foi avaliar a prevalência do uso das diversas formas de tabaco e o perfil demográfico das mulheres fumantes no Estado do Paraná. A caracterização das mulheres em diferentes regiões do estado e sua relação com o uso do tabaco são essenciais para melhor compreensão desta situação e para o planejamento e monitoramento de intervenções.

\section{Método}

Este estudo caracterizou-se como transversal de base populacional, realizado com mulheres residentes em sete cidades do Paraná, representando três macrorregiões do estado: norte (Londrina, Cambé, Maringá), sul (Curitiba, Irati) e sudoeste (Cascavel, Pato Branco). A amostra foi calcuada por meio de amostragem probabilística por cluster. Foi realizada a identificação dos setores censitários na zona urbana de cada cidade, sendo excluídos os setores censitários com menos de vinte domicílios permanentes. Selecionaram-se aleatoriamente 15 setores censitários (clusters) por cidade e o primeiro domicílio dentro deste setor foi considerado o ponto geográfico central. Caso houvesse uma pessoa elegível para o estudo na residência, este foi o primeiro domicílio, caso não houvesse, selecionaram-se os domicílios em sequência à direita, à esquerda, à frente e atrás até que vinte entrevistas fossem completadas no setor censitário. As cidades foram selecionadas por conveniência, porém, elas são representativas da população do estado.

Para a coleta de dados utilizaram-se as questões incluídas na Pesquisa Especial de Tabagismo 
(PETab), realizada no Brasil em 2008, como parte da Pesquisa Nacional por Amostra de Domicílios (PNAD) ${ }^{12}$. Esta pesquisa foi uma adaptação da Global Adult Tobacco Survey (GATS) que segue um modelo global para o monitoramento sistemático do uso de tabaco em vários países. A coleta de dados abrangeu diversos fatores relacionados ao uso do tabaco, tais como: local de compra, mídia associada ao tabaco e exposição ambiental à fumaça do tabaco. Porém este artigo se limita aos dados de prevalência das diversas formas de tabaco e perfil demográfico das fumantes.

Todas as questões utilizadas neste artigo foram idênticas às questões utilizadas na PETab para facilitar comparações com dados obtidos no nível nacional. A técnica empregada na coleta de dados foi de entrevistas individuais facea-face. Os dados foram coletados por meio da tecnologia CAPI (Computer Assisted Personal Interviews), que utilizou como instrumento um questionário digitalizado em computador portátil. Todas as entrevistadoras eram do sexo feminino e as entrevistas foram realizadas aos sábados e domingos.

Em cada domicílio, somente uma residente com idade $\geq 18$ anos foi selecionada para responder o questionário. Caso houvesse mais de uma mulher adulta no domicílio no momento da entrevista, a que possuísse a data de aniversário mais próximo da data da entrevista era selecionada.

A pesquisa foi realizada para atingir uma amostra final de 2.100, com entrevistas finais válidas completas, com seleção probabilística e erro amostral total de $2 \%$ e $6 \%$ por segmento. As margens de erro amostral foram calculadas a partir de uma estimativa da população de cada município, com base nas informações disponíveis do Instituto Brasileiro de Geografia e Estatística (IBGE) 31. O intervalo de confiança foi de $95 \%$ (IC95\%) e o grau de homogeneidade de opiniões da população estimado (split) foi de $50 \%$ x 50\%, uma vez que a população estudada foi considerada heterogênea.

\section{Análise estatística}

A análise dos dados foi realizada com a versão 17.0 do SPSS (SPSS Inc., Chicago, Estados Unidos) e a versão 9.2 do SAS (SAS Inst., Cary, Estados Unidos). Os dados foram apresentados descritivamente com base nos números absolutos, percentuais e médias. A associação entre características da população e o uso de produtos derivados do tabaco foi analisada por meio de comparações bivariadas utilizando os testes quiquadrado e teste t para as variáveis categóricas e contínuas, respectivamente. A regressão logística multivariada foi utilizada para avaliar a associação entre as características da população e o uso de produtos derivados do tabaco. Também foram avaliadas as razões de chance (odds ratio - OR) e IC95\%.

Para a análise de comparações realizamos alguns agrupamentos de variáveis - (1) escolaridade foi classificada em três categorias: Ensino Fundamental completo ou incompleto, Ensino Médio completo ou incompleto e Ensino Superior completo ou incompleto; (2) embora utilizássemos a classificação do IBGE para ocupação 32 , agrupamos as categorias em: (a) técnico/profissional (membros superiores do poder público, dirigentes de organizações, profissionais das ciências e das artes, técnicos de nível médio); (b) trabalhadoras em serviços administrativos; (c) "ocupações elementares" (trabalhadoras em serviços, vendedoras do comércio em lojas e mercados, trabalhadoras agropecuárias, florestais, da caça e pesca, trabalhadoras da produção de bens e serviços industriais, trabalhadoras de manutenção e reparação); (d) donas de casa; (e) aposentadas; (f) estudantes; e (g) desempregadas; (3) em relação a estado civil, agrupamos as categorias de separada e divorciada, resultando em cinco categorias: (a) solteira, (b) casada; (c) em união estável; (d) separada/divorciada; e (e) viúva; (4) a renda familiar mensal foi calculada dividindo a renda familiar mensal pelo número de pessoas sustentadas por esta renda.

Oprotocolo de pesquisa deste estudo foi aprovado pelo Comitê de Ética em Pesquisa da University of Alabama at Birmingham (X070813006), da Pontifícia Universidade Católica do Paraná (3004) e Comissão Nacional de Ética em Pesquisa (CONEP; 14651) e seguiu os princípios éticos contidos na Declaração de Helsinki.

\section{Resultados}

\section{Descrição da amostra}

A amostra foi composta por 2.153 mulheres, sendo aproximadamente 300 participantes por cidade. A média de idade foi de 45,5 anos (desviopadrão - DP = 16,0). A maioria das entrevistadas $(65,7 \%)$ era casada, tinha filhos $(83,1 \%)$ e era dona de casa (43,8\%). Em relação à escolaridade, 5,1\% das participantes nunca frequentaram a escola e 47,3\% completaram o Ensino Médio. A renda per capita familiar de até um salário mínimo foi predominante (54\%). As características da amostra total são apresentadas na Tabela 1. A prevalência do uso do tabaco fumado na amostra total foi de $13,4 \%$, sendo que $12,3 \%$ fumavam diariamente. A média de idade das fumantes foi de 45,1 anos 
Perfil da amostra de mulheres. Paraná, Brasil, 2010.

\begin{tabular}{|c|c|c|}
\hline Variável & $\begin{array}{l}\text { Amostra total }(\mathrm{N}=2.153) \\
\qquad \mathrm{n}(\%)\end{array}$ & $\begin{array}{l}\text { Usuárias de tabaco fumado }(\mathrm{n}=289) \\
\mathrm{n}(\%)\end{array}$ \\
\hline \multicolumn{3}{|l|}{ Idade (anos) } \\
\hline $18-24$ & $216(10,1)$ & $17(5,9)$ \\
\hline $25-44$ & $822(38,3)$ & $115(39,9)$ \\
\hline $45-64$ & $810(37,8)$ & $135(46,9)$ \\
\hline$\geq 65$ & $297(13,9)$ & $21(7,3)$ \\
\hline \multicolumn{3}{|l|}{ Escolaridade } \\
\hline Não frequentaram & $109(5,1)$ & $10(3,5)$ \\
\hline Ensino Fundamental incompleto & $670(31,3)$ & $114(40,0)$ \\
\hline Ensino Fundamental completo & $249(11,6)$ & $40(14,0)$ \\
\hline Ensino Médio incompleto & $92(4,3)$ & $14(4,9)$ \\
\hline Ensino Médio completo & $634(29,6)$ & $81(28,1)$ \\
\hline Ensino Superior incompleto & $105(4,9)$ & $9(3,1)$ \\
\hline Ensino Superior completo & $280(13,1)$ & $17(5,9)$ \\
\hline \multicolumn{3}{|l|}{ Estado civil } \\
\hline Solteira & $308(14,3)$ & $52(18,0)$ \\
\hline Casada & $1.415(65,7)$ & $163(56,6)$ \\
\hline União estável & $46(2,1)$ & $18(6,3)$ \\
\hline Divorciada/Separada & $155(7,2)$ & $28(9,8)$ \\
\hline Viúva & $229(10,6)$ & $27(9,4)$ \\
\hline \multicolumn{3}{|l|}{ Possui filhos } \\
\hline Sim & $1.790(83,1)$ & $254(88,2)$ \\
\hline Não & $363(16,9)$ & $34(11,8)$ \\
\hline \multicolumn{3}{|l|}{ Renda familiar/número de pessoas dependentes desta renda } \\
\hline \multicolumn{3}{|l|}{ (em Reais) } \\
\hline Até 127,50 & $25(1,5)$ & $6(2,7)$ \\
\hline $127,51-255,00$ & $225(13,1)$ & $48(21,3)$ \\
\hline $255,01-510,00$ & $675(39,4)$ & $74(32,9)$ \\
\hline $510,01-1.020,00$ & $552(32,2)$ & $71(31,6)$ \\
\hline Acima de 1.020,00 & $235(13,7)$ & $26(11,6)$ \\
\hline \multicolumn{3}{|l|}{ Ocupação } \\
\hline $\begin{array}{l}\text { Membros superiores do poder público, dirigentes de } \\
\text { organizações }\end{array}$ & $59(2,8)$ & $5(1,8)$ \\
\hline Profissionais das ciências e das artes & $190(9,0)$ & $12(4,3)$ \\
\hline Técnicas de nível médio & $79(3,8)$ & $13(4,6)$ \\
\hline Trabalhadoras de serviços administrativos & $114(5,4)$ & $11(3,9)$ \\
\hline $\begin{array}{l}\text { Trabalhadoras dos serviços, vendedores do comércio em lojas e } \\
\text { mercados }\end{array}$ & $461(21,9)$ & $99(35,1)$ \\
\hline Trabalhadoras agropecuárias, florestais, da caça e pesca & $4(0,2)$ & $1(0,4)$ \\
\hline Trabalhadoras da produção de bens e serviços industriais & $134(6,4)$ & $13(4,6)$ \\
\hline Donas de casa & $923(43,8)$ & $117(41,3)$ \\
\hline Aposentadas & $92(4,4)$ & $10(3,5)$ \\
\hline Estudantes & $45(2,1)$ & $2(0,7)$ \\
\hline Desempregadas & $6(0,3)$ & $0(0,0)$ \\
\hline
\end{tabular}


(DP = 13,1). A média de idade declarada do início do hábito tabágico foi 17,0 anos ( $\mathrm{DP}=6,9$ ). A maioria das fumantes tinha uma renda per capita familiar de até um salário mínimo (56,9\%). Em relação à escolaridade, $37,1 \%$ completaram o Ensino Médio, sendo que dentre estas, 5,9\% completaram o Ensino Superior.

De acordo com o que pode ser observado na Tabela 2, a prevalência do uso do tabaco fumado distribuída entre as cidades foi $12 \%$ em Cambé, $10 \%$ em Cascavel, 17,1\% em Curitiba, 19\% em Irati, 11,7\% em Londrina, 10,6\% em Maringá e $13,5 \%$ em Pato Branco. O consumo de tabaco diário foi observado em 91,7\% desta população e o cigarro industrializado foi o produto mais consumido (93,8\%). Nesta amostra, as usuárias de narguilé $(0,2 \%)$ não faziam uso de outro produto derivado do tabaco, enquanto que 56,7\% das mulheres que consumiam cigarro de palha faziam uso concomitante do cigarro industrializado. Nenhuma participante indicou uso de cigarros de cravo ou de Bali, bidis, cachimbo ou charuto. Somente três participantes indicaram uso de tabaco de mascar. Nenhuma participante indicou o uso de outros produtos derivados do tabaco sem fumaça.

Dentre as fumantes diárias de cigarros industrializados, a média de número de cigarros fumados por dia foi de 10,2 (DP = 7,0); 75\% das fumantes diárias de cigarros industrializados indicaram fumar 10 ou menos cigarros por dia, o que as classificariam como "fumantes leves". Porém, $18,9 \%$ das fumantes diárias de cigarros industrializados afirmaram fumar o seu primeiro cigarro dentro de cinco minutos depois de acordar e $37,4 \%$ dentro de 6 a 10 minutos, indicando uma dependência da nicotina relativamente alta.

O maior percentual de fumantes foi encontrado na faixa etária de 45 e 64 anos (46,9\%). Observou-se que as mulheres com mais de 50 anos iniciaram o consumo do tabaco fumado em média 2,1 anos mais tarde que as mulheres até 50 anos. Em relação à cessação, $49 \%$ tentaram parar de fumar nos últimos 12 meses. A maioria das participantes indicou que tem a intenção de parar de fumar nos próximos 12 meses $(56,7 \%)$.

\section{Comparações}

Cerca de $15 \%(14,4 \%)$ das participantes indicaram serem ex-fumantes. Com o objetivo de obter um perfil das fumantes comparadas às não fumantes, as ex-fumantes foram retiradas da análise, resultando em uma amostra final de 1.885 participantes.

Foram observadas associações significativas entre cidade, estado civil, escolaridade, ocupação e o hábito de fumar ( $\mathrm{p}=0,0003 ; \mathrm{p}<0,0001$; $\mathrm{p}<0,001$ e $\mathrm{p}<0,0001$, respectivamente) entre fumantes e não fumantes. Mulheres residentes em Irati (20,4\% vs. 11,8\%), mulheres que completaram até a quarta série primária ou menos (57,7\% vs. 45\%), mulheres em "ocupações elementares" (39,9\% vs. $26 \%)$ foram mais propensas a serem fumantes do que não fumantes. Por outro lado, mulheres casadas foram mais propensas a nunca fumarem (68,3\% vs. 56,8\%).

A Tabela 3 apresenta os resultados da análise multivariada. Mulheres casadas ou viúvas foram menos propensas a serem fumantes, comparadas

Tabela 2

Distribuição de prevalência do uso do tabaco em mulheres, em sete cidades do Paraná, Brasil.

\begin{tabular}{|c|c|c|c|c|}
\hline Cidades & $\begin{array}{c}\text { Prevalência do uso do } \\
\text { tabaco fumado } \\
\text { n (\%) }\end{array}$ & $\begin{array}{c}\text { Cigarro industrializado } \\
\star \\
n(\%)\end{array}$ & Cigarro de palha * & Narguilé * \\
\hline Cascavel & $31(10,0)$ & $30(96,8)$ & $0(0,0)$ & $1(3,2)$ \\
\hline Maringá & $33(10,6)$ & $31(93,9)$ & $1(3,0)$ & $2(6,1)$ \\
\hline Londrina & $36(11,7)$ & $35(97,2)$ & $3(8,3)$ & $0(0,0)$ \\
\hline Cambé & $36(12,0)$ & $34(94,4)$ & $4(11,4)$ & $0(0,0)$ \\
\hline Irati & $59(19,0)$ & $50(84,7)$ & $19(32,2)$ & $1(1,7)$ \\
\hline Pato Branco & $41(13,5)$ & $40(97,6)$ & $2(3,8)$ & $0(0,0)$ \\
\hline Curitiba & $53(17,1)$ & $51(96,2)$ & $3(5,7)$ & $1(1,9)$ \\
\hline Total & $289(100,0)$ & $271(93,8)$ & $30(10,4)$ & $5(1,7)$ \\
\hline
\end{tabular}

* Dentre as fumantes, estas porcentagens se referem às porcentagens de fumantes que fumam estes produtos. 
Odds ratio (OR) e intervalos de 95\% de confiança (IC95\%) nas comparações entre fumantes e não fumantes.

\begin{tabular}{|c|c|c|}
\hline Variável & OR (IC95\%) & Valor de $p$ \\
\hline Idade & $1,00(0,99-1,02)$ & 0,75 \\
\hline \multicolumn{3}{|l|}{ Cidade } \\
\hline Cascavel (vs. Cambé) & $0,89(0,49-1,59)$ & 0,68 \\
\hline Curitiba (vs.Cambé) & $1,46(0,84-2,53)$ & 0,18 \\
\hline Irati (vs. Cambé) & $2,08(1,22-3,54)$ & 0,0073 \\
\hline Londrina (vs. Cambé) & $0,91(0,51-1,63)$ & 0,76 \\
\hline Maringá (vs. Cambé) & $1,03(0,58-1,84)$ & 0,92 \\
\hline Pato Branco (vs. Cambé) & $1,29(0,73-2,28)$ & 0,38 \\
\hline \multicolumn{3}{|l|}{ Estado civil } \\
\hline Casadas (vs. solteiras) & $0,47(0,30-0,73)$ & 0,0008 \\
\hline União estável (vs. solteiras) & $2,49(1,12-5,53)$ & 0,0249 \\
\hline Separadas/Divorciadas (vs. solteiras) & $0,90(0,47-1,71)$ & 0,74 \\
\hline Viúvas (vs. solteiras) & $0,43(0,22-0,87)$ & 0,0186 \\
\hline \multicolumn{3}{|l|}{ Escolaridade } \\
\hline $\begin{array}{l}\text { Ensino Médio completo ou incompleto (vs. primeiro } \\
\text { grau ou menos) }\end{array}$ & $0,74(0,51-1,07)$ & 0,11 \\
\hline $\begin{array}{l}\text { Ensino Superior completo ou incompleto (vs. primeiro grau ou } \\
\text { menos) }\end{array}$ & $0,41(0,22-0,87)$ & 0,0064 \\
\hline \multicolumn{3}{|l|}{ Ocupação } \\
\hline Trabalhadoras de serviços administrativos (vs. técnico/profissional) & $0,78(0,32-1,90)$ & 0,58 \\
\hline "Ocupações elementares" (vs. técnico/profissional) & $1,47(0,81-2,68)$ & 0,2 \\
\hline Donas de casa (vs. técnico/profissional) & $1,00(0,55-1,82)$ & 0,99 \\
\hline Aposentadas (vs. técnico/profissional) & $1,02(0,39-2,66)$ & 0,96 \\
\hline Estudantes (vs. técnico/profissional) & $0,20(0,02-1,58)$ & 0,13 \\
\hline Desempregadas (vs. técnico/profissional) & - & - \\
\hline Renda familiar mensal/pessoas sustentadas por esta renda & $1,00(1,0-1,0)$ & 0,57 \\
\hline
\end{tabular}

a mulheres solteiras (OR = 0,47; IC95\%: 0,30-0,73 e OR = 0,43; IC95\%: 0,22-0,87, respectivamente). Mulheres vivendo em condição de uniões estáveis foram mais propensas a serem fumantes que mulheres solteiras (OR = 2,49; IC95\%: 1,12-5,53). Da mesma forma, mulheres com educação de nível superior (mesmo que não completos) foram menos propensas a serem fumantes do que mulheres com Ensino Fundamental ou menos (OR = 0,41; IC95\%: 0,22-0,87). Nenhuma associação significativa foi encontrada entre idade ou renda familiar mensal e o fato de serem fumantes.

\section{Discussão}

Para promover o controle do tabaco, os governos ao redor do mundo têm instituído medidas de saúde pública 1,33. Apesar disto, a indústria do tabaco tem utilizado várias estratégias na tentativa de estimular seu consumo 33,34. Uma das estratégias utilizadas por essas empresas é o marketing focando as mulheres dos países em desenvolvimento, tendo em vista que esse público não costumava consumir tabaco 34 .

No Brasil, os estudos de prevalência do uso do tabaco têm apontado queda no número de homens e mulheres fumantes, porém as mulheres apresentam maior dificuldade em parar de consumir o tabaco, bem como iniciam mais precocemente o hábito de fumar 35 . A prevalência de uso de tabaco $(13,4 \%)$ no presente estudo foi similar à prevalência do uso de tabaco entre mulheres brasileiras (13,3\%) e mulheres do Sul do Brasil (15,9\%), descritas pela PETab (2008) 12. Na presente amostra $74,1 \%$ das mulheres nunca fumaram, valor levemente superior aos achados do PETab $(71,7 \%)$ 12. Esse achado está de acordo com a tendência de queda do consumo de tabaco no Brasil. Porém, houve uma variação considerável na prevalência de fumantes entre as cidades, com Irati tendo a maior prevalência 
de fumantes. Também foi nesta região onde o uso do cigarro de palha foi maior. Isso pode ser devido ao fato de esta região ser produtora de tabaco. Estes dados confirmam a necessidade de estudos regionais para que intervenções pontuais sejam desenvolvidas.

Em relação aos fatores demográficos, os resultados confirmam os achados de outras pesquisas onde já foi demonstrada a associação entre escolaridade e o uso do tabaco 36,37. Mulheres com baixa escolaridade tiveram maior probabilidade de serem fumantes que mulheres com curso superior (mesmo que incompleto). Estes resultados demonstram que maiores esforços devem ser feitos para promover a prevenção e cessação em mulheres com baixa escolaridade. Embora renda familiar e ocupação tenham demonstrado relação com o uso do tabaco, estas relações desapareceram na análise multivariada, com escolaridade e estado civil sendo as variáveis demográficas mais importantes. Em relação a estado civil associações importantes emergiram deste estudo. Enquanto mulheres casadas e viúvas foram menos propensas a serem fumantes que mulheres solteiras. Mulheres vivendo em condição de união estável foram mais propensas a serem fumantes que mulheres solteiras.

Estes resultados em parte reforçam estudos anteriores os quais, explorando o impacto do estado civil no uso de tabaco por mulheres em diferentes contextos culturais, evidencia que mulheres solteiras ou divorciadas são mais propensas a fumar, comparado com mulheres casadas ou vivendo uniões estáveis 38,39,40. Entretanto, a convivência em união estável neste estudo demonstrou ser significativamente impactante na probabilidade de uso de tabaco por mulheres. Maior aprofundamento sobre aspectos como suporte social, conflitos e segurança relacional no contexto de estados civis considerados aqui como uniões estáveis, podem oportunizar melhor compreensão dos resultados. No nosso estudo não foram encontrados usuárias de cigarro de cravo ou de Bali, cachimbo, charuto ou bidi, e o consumo de narguilé também foi baixo. O consumo de cigarro industrializado foi maior que as outras formas de tabaco $(12,6 \%)$, bem como o percentual de mulheres que fumam diariamente $(91,7 \%)$ em relação às que fumam ocasionalmente $(8,3 \%)$, achados que estão de acordo com outros estudos $8,36,41$.

Segundo Fagerström 42, o tempo de espera entre acordar e fumar o primeiro cigarro é uma medida confiável de dependência do tabaco, e esta medida vem sendo utilizada em vários estudos com esse objetivo. Embora 2/3 das participantes indicaram fumar dez ou menos cigarros ao dia, elas demonstraram uma dependência da nicotina relativamente alta. Na presente amostra, 18,9\% fumam dentro de cinco minutos após despertar pela manhã e 37,4\% dentro de dez minutos. De fato, Baker et al. 43 afirmaram que fumantes que esperam entre 5 e 30 minutos para iniciar o consumo de tabaco tendem a voltar a consumi-lo de forma esporádica apenas um dia depois de decidir parar e em 21 dias o hábito tende a retornar ao que era anteriormente.

Quando foi analisado o uso de tabaco e sua distribuição por faixa etária, observou-se que a maior prevalência de fumantes estava na faixa etária entre 45 e 64 anos de idade. Também se observou que as mulheres com mais de 50 anos iniciaram o consumo do tabaco fumado em média 2,1 anos mais tarde que as mulheres com menos de 50 anos de idade. Estes resultados sugerem que as mulheres mais jovens estão iniciando o uso do tabaco mais cedo que mulheres mais velhas. Visto os resultados da iniciação precoce encontrados por outros pesquisadores, há necessidade de estudos prospectivos para melhor entender a iniciação do uso do tabaco em mulheres 9,16,17.

Benefícios advindos da cessação do hábito de consumir tabaco já estão bem descritos na literatura como destaque para a redução do risco de desenvolver doenças relacionadas ao consumo do tabaco e mortalidade. Nesta amostra, $49 \%$ das fumantes indicaram que tentaram parar de fumar nos últimos 12 meses, índice maior que o encontrado em uma pesquisa anterior em Curitiba $(42,4 \%){ }^{9}$. Além disto, $56,7 \%$ das fumantes afirmaram planejar parar de fumar nos próximos meses. Estes resultados indicam uma motivação relativamente alta para a cessação do uso do tabaco. Futuras pesquisas são necessárias para entender melhor os fatores relacionados ao sucesso e recaída destas mulheres em programas de cessação.

Os resultados obtidos neste estudo possuem algumas limitações. Primeiro, o delineamento transversal impossibilita verificar a relação de causalidade. Segundo, os dados desse estudo se basearam em informações fornecidas pelas entrevistadas e podem estar super ou subestimados. Terceiro, a escolha das cidades, embora representativa da população do estado, foi feita por conveniência.

A partir dos resultados encontrados no presente estudo, conclui-se que houve diminuição na prevalência do uso do tabaco em mulheres no Paraná, quando comparado com resultados de estudos anteriores. Porém, há uma disparidade em prevalência de fumantes entre as cidades. Escolaridade e estado civil são fatores extremamente importantes a serem considerados no desenvolvimento de programas visando o controle 
do tabaco em mulheres. Neste sentido, faz-se necessária intervenção direcionada para subpopulações de mulheres dentro do Paraná, com fins de reverter o quadro relatado. Além disto, futuras pesquisas são necessárias a fim de construir maior conhecimento sobre as mulheres usuárias de tabaco no Paraná e políticas públicas voltadas para essa população.

\section{Resumo}

O objetivo deste estudo foi avaliar a prevalência do uso de produtos derivados do tabaco e o perfil demográfico de mulheres fumantes no Paraná, Brasil. Foi um estudo transversal de base populacional com amostragem por cluster envolvendo 2.153 mulheres com 18 anos de idade ou mais em sete cidades. A prevalência do uso do tabaco fumado foi de 13,4\% variando de $10 \%$ (Cascavel) a 19\% (Irati). Na análise multivariada, cidade, estado civil e escolaridade foram significativamente associados ao uso do tabaco. Mulheres em Irati $(O R=$ 2,08; IC95\%: 1,22-3,54) foram mais propensas a serem fumantes que mulheres em Cambé. As casadas ou viúvas foram menos propensas a serem fumantes $(O R=$ 0,47; IC95\%: 0,30-0,73 e OR = 0,43; IC95\%: 0,22-0,87) que as solteiras. Mulheres em união estável foram mais propensas a serem fumantes que as solteiras $(O R=$ 2,49; IC95\%: 1,12-5,53) e mulheres com curso superior foram menos propensas a serem fumantes que as com primeiro grau ou menos (OR = 0,41; IC95\%: 0,22-0,87). Estes resultados confirmam a necessidade de programas de controle do tabaco que levem em consideração as questões de gênero e regiões.

Tabaco; Tabagismo; Mulheres

\section{Colaboradores}

A concepção e a organização do artigo foram feitas com a participação de todos os autores. I. C. Scarinci e R. C. Cruz foram responsáveis pela formulação e elaboração do projeto, incluindo o desenho da pesquisa e elaboração do instrumento de coleta de dados em colaboração com S. Person. I. C. Scarinci e R. C. Cruz também foram responsáveis pelo treinamento e supervisão da coleta de dados e desenvolvimento e supervisão deste artigo. L. Bittencourt responsabilizou-se pela revisão bibliográfica e desenvolveu a primeira versão do artigo. S. Person foi responsável pela análise dos dados, interpretação e apresentação dos resultados e deu toda assistência na discussão dos resultados. S. T. Moysés colaborou com a revisão do conteúdo do texto.

\section{Agradecimentos}

O projeto obteve financiamento junto ao Instituto $\mathrm{Na}$ cional de Saúde Americano (RO1 DA 24875).

\section{Referências}

1. World Health Organization. WHO report on the global tobacco epidemic, 2008: the MPOWER package. Geneva: World Health Organization; 2008.

2. Mokdad AH, Marks JS, Stroup DF, Gerberding JL. Actual causes of death in the United States, 2000. JAMA 2004; 291:1238-45.

3. Machado A, Nicolau R, Dias CM. Consumo de tabaco na população portuguesa retratado pelo Inquérito Nacional de Saúde (2005/2006). Rev Port Pneumol 2009; 15:1005-27.

4. Lopez AD, Mathers CD, Ezzati M, Jamison DT, Murray CJ. Global and regional burden of disease and risk factors, 2001: systematic analysis of population health data. Lancet 2006; 367:1747-57.

5. Hodgson TA, Meiners MR. Cost-of-illness methodology: a guide to current practices and and procedures. Milbank Mem Fund Q 1982; 60:429-62.

6. Pinto M, Ugá MAD. Os custos de doenças tabacorelacionadas para o Sistema Único de Saúde. Cad Saúde Pública 2010; 26:1234-45.

7. Cavalcante TM. O controle do tabagismo no Brasil: avanços e desafios. Rev Psiquiatr Clín 2005; 32: 283-300.

8. Leitão Filho FS, Galduróz JCF, Noto AR, Nappo AS, Carlini EA, Nascimento AO, et al. Levantamento randomizado sobre a prevalência e tabagismo nos maiores municípios do Brasil. J Bras Pneumol 2009; 35:1204-11.

9. Instituto Nacional de Câncer. Inquérito domiciliar sobre comportamentos de risco e morbidade referida de doenças e agravos não transmissíveis: Brasil, 15 capitais e Distrito Federal, 2002-2003. Rio de Janeiro: Instituto Nacional de Câncer; 2004.

10. Bialous SA, Presman S, Gigliotti A, Muggli M, Hurt R. A resposta da indústria do tabaco à criação de espaços livres de fumo no Brasil. Rev Panam Salud Pública 2010; 27:283-90.

11. Ministério da Saúde. Legislação federal de controle do tabagismo. Rio de Janeiro: Ministério da Saúde; 2004.

12. Instituto Nacional de Câncer/Organização PanAmericana da Saúde. Pesquisa especial de Tabagismo - PETab: relatório Brasil. Rio de Janeiro: Instituto Nacional de Câncer; 2011.

13. Associação Brasileira da Indústria do Fumo. Produção de fumo. http://www.abifumo.org.br/pro du.htm (acessado em 27/Out/2011). 
14. Monteiro CA, Cavalcanti TM, Moura EC, Claro RM, Szwarcwald CL. Population-based evidence of a strong decline in prevalence of smokers in Brazil (1989-2003). Bull World Organ 2007; 85:527-34.

15. Dim Iglesias R, Jha P, Pinto M, Costa-e-Silva VL, Godinho J. Controle do tabagismo no Brasil. Washington DC: World Bank; 2007.

16. Instituto Nacional de Câncer. Vigescola. Vigilância de tabagismo em escolares: dados e fatos de 12 capitais brasileiras. Rio de Janeiro: Instituto Nacional de Câncer; 2004.

17. Galduróz JCF, Noto AR, Nappo SA, Carlini EA. Household survey on drug abuse in Brazil: Study involving the 107 major cities of the country 2001. Addict Behav 2005; 30:545-56.

18. Stockton MC, McMahon SD, Jason LA. Gender and smoking behavior in a worksite smoking cessation program. Addict Behav 2000; 25:347-60.

19. Osler M, Prescott E, Godtfredsen N, Hein HO, Schnohr P. Gender and determinants of smoking cessation: a longitudinal study. Prev Med 1999; 29:57-62.

20. Wetter DW, Kenford SL, Smith SS, Fiore MC, Jorenby DE, Baker TB. Gender differences in smoking cessation. J Consult Clin Psychol 1999; 67:555-62.

21. Bohadana A, Nilsson F, Rasmussen T, Martinet Y. Gender differences in quit rates following smoking cessation with combination nicotine therapy: influence of baseline smoking behavior. Nicotine Tob Res 2003; 5:111-6.

22. Caraballo RS, Giovino GA, Pechacek TF, Mowery PD, Richter PA, Strauss WJ, et al. Racial and ethnic differences in serum cotinine levels of cigarette smokers: Third National Health and Nutrition Examination Survey, 1988-1991. JAMA 1998; 280:135-9.

23. Collins BN, Wileyto EP, Patterson F, Rukstalis M, Audrain-McGovern J, Kaufmann V, et al. Gender differences in smoking cessation in a placebo-controlled trial of bupropion with behavioral counseling. Nicotine Tob Res 2004; 6:27-37.

24. Garrett BE, Dube SR, Trosclair A, Caraballo RS, Pechacek TF. Cigarette smoking - United States, 1965-2008. MMWR Surveill Summ 2011; 60 Suppl:109-13.

25. Croghan IT, Ebert JO, Hurt RD, Hays JT, Dale LC, Warner N, et al. Gender differences among smokers receiving interventions for tobacco dependence in a medical setting. Addict Behav 2009; 34:61-7.

26. Castro MR, Matsuo T, Nunes SOV. Characteristics of smokers smoking cessation interventions: an analysis of sex differences. Addict Disord Their Treat 2010; 9:135-42.

27. Mackay J, Ericksen M, Shafey O. The tobacco atlas. $2^{\text {nd }}$ Ed. Atlanta: American Cancer Society; 2006.

28. Bialous SA, Shatenstein S. Profits over people: tobacco industry activities to market cigarettes and undermine public health in Latin America and the Caribbean. Washington DC: Pan-American Health Organization; 2002.
29. Greaves L, Jategaonkar N, Sanchez S. Turning a new leaf: women, tobacco, and the future. British Columbia Centre of Excellence for Women's Health and International Network of Women Against Tobacco. Vancouver: British Columbia Centre of Excellence for Women's Health; 2006.

30. Bialous SA, Presman S, Gigliotti A, Muggli M, Hurt R. A resposta da indústria do tabaco à criação de espaços livres de fumo no Brasil. Rev Panam Salud Pública 2010; 27:283-90.

31. Instituto Brasileiro de Geografia e Estatística. Pesquisa Nacional por Amostra de Domicílios. http:// www.ibge.gov.br/estatistica/populacao/trabalho erendimento/pnad2008/suplementos/tabagis mo/default.shtm (acessado em 09/Set/2011).

32. Instituto Brasileiro de Geografia e Estatística. Classificação de Ocupações para Pesquisas Domiciliares - COD: principais diferenças entre a COD e a CBO-domiciliar. http://www.ibge.gov.br/home/ estatistica/indicadores/sipd/oitavo_forum/COD. pdf (acessado em 20/Set/2011).

33. Bialous SA, Fox BJ, Glantz SA. Tobacco industry allegations of "illegal lobbying" and state tobacco control. Am J Public Health 2001; 91:62-7.

34. McDaniel PA, Malone RE. Creating the "desired mindset": Philip Morris's efforts to improve its corporate image among women. Women Health 2009; 49:441-74.

35. Peixoto SV, Firmo JO, Lima-Costa MA. Fatores associados ao índice de cessação do hábito de fumar em duas diferentes populações adultas (Projetos Bambuí e Belo Horizonte). Cad Saúde Pública 2007; 23:1319-28.

36. Silva GA, Valente JG, Almeida LM, Moura EC, Malta DC. Tabagismo e escolaridade no Brasil, 2006. Rev Saúde Pública 2009; 43 Suppl 2:48-56.

37. Zanini RR, Moraes AB, Trindade AC, Riboldi J, Medeiros LR. Prevalência e fatores associados ao consumo de cigarros entre estudantes de escolas estaduais do ensino médio de Santa Maria, Rio Grande do Sul, Brasil, 2002. Cad Saúde Pública 2006; 22:1619-27.

38. Lindström M. Social capital, economic conditions, marital status and daily smoking: a populationbased study. Public Health 2010; 124:71-7.

39. Freire K, Padilha PC, Saunders C. Fatores associados ao uso de álcool e cigarro na gestação. Rev Bras Ginecol Obstet 2009; 31:335-41.

40. Cho HJ, Khang YH, Jun HJ, Kawachi I. Marital status and smoking in Korea: the influence of gender and age. Soc Sci Med 2008; 66:609-19.

41. Viegas CA. Formas não habituais de uso do tabaco. J Bras Pneumol 2008; 34:1069-73.

42. Fagerström KO. Measuring degree of physical dependence to tobacco smoking with reference to individualization of treatment. Addict Behav1978; 5:235-41.

43. Baker TB, Piper ME, McCarthy DE, Bolt DM, Smith SS, Kim S-Y, et al. Time to first cigarette in the morning as an index of ability to quit smoking: implications for nicotine dependence. Nicotine Tob Res 2007; 9 Suppl 4:S555-70.

Recebido em 16/Nov/2011

Versão final reapresentada em 01/Mai/2012

Aprovado em 09/Mai/2012 